\title{
Exciting the optical response of nanowire metamaterial films on the tip of optical fibres
}

\author{
I. T. Leite', A. Hierro-Rodríguez ${ }^{1,2}$, A. O. Silva ${ }^{1,3,4}{ }^{4}$, J. M. Teixeira², C. T. Sousa², M. P. Fernández-García ${ }^{2}$, \\ J. P. Araújo ${ }^{2,5}$, P. A. S. Jorge', and A. Guerreiro" ${ }^{*, 5}$ \\ ${ }^{1}$ INESC TEC (coordinated by INESC Porto), Rua Campo Alegre, 4169-007 Porto, Portugal \\ ${ }^{2}$ IFIMUP and IN - Institute of Nanoscience and Nanotechnology, Rua Campo Alegre, 4169-007 Porto, Portugal \\ ${ }^{3}$ LEA (Applied Electromagnetism Laboratory), C.U. José da Silveira Neto, Belém, Brazil \\ ${ }^{4}$ Universidade Federal do Pará, C.U. José da Silveira Neto, Belém, Brazil \\ ${ }^{5}$ Departamento de Física e Astronomia, Faculdade de Ciências, Universidade do Porto, Rua Campo Alegre, 4169-007 Porto, Portugal
}

Received 19 June 2013, revised 17 July 2013, accepted 25 July 2013

Published online 7 August 2013

Keywords nanowire metamaterials, surface plasmon-polaritons, fibre optics

* Corresponding author: e-mail ariel@fc.up.pt, Phone: +351 220402 301, Fax: +351 220402437

This Letter reports on the assembly on the tip of an optical fibre of a metamaterial film fabricated by a self-assembly bottom-up method, composed of silver nanowires embedded in an alumina matrix. By illuminating the film through the fibre in a reflection configuration, we observe experimentally the optical response of the metamaterial in agreement with theoretical predictions and interpreted as the excitation of surface plasmon-polaritons in the cylindrical surface of the nanowires. These results pave the way for low-cost optical fibre devices that incorporate metamaterial films.

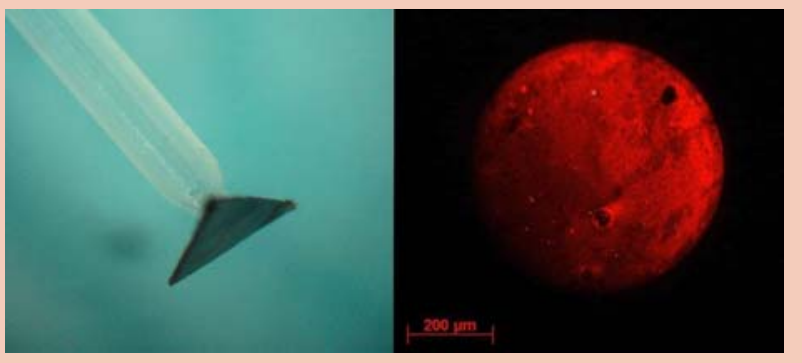

Micrographs of a nanowire metamaterial film mounted on the tip of an optical fibre (left) and top-view of the sample in a transmission configuration (right). The red colour results from the absorption of the other optical wavelengths by the plasmon modes in the metamaterial.
1 Introduction The coupling between light and surface plasmon-polaritons (SPPs) in thin metallic films is very sensitive to variations of the optical properties of the surrounding medium $[1,2]$ and is extensively used as the sensing principle for a wide range of detecting and measuring devices, including optical fibre based sensors [3, 4]. Only light modes with electric field components perpendicular to the surface of the metal can excite SPPs and therefore most of the fibre based sensors are adaptations of the Kretschmann configuration [5] consisting of a thin metallic film deposited in a side-polished or tapered fibre [6, 7]. The challenge in developing plasmonic sensors using metallic films deposited on the tip of a cleaved fibre is that the electric field of the guided modes in the fibre is mostly parallel to the surface of the metal, and therefore unable to excite SPPs. To circumvent this difficulty, other proposals have replaced the thin film with metallic nanostructures with geometries capable of supporting SPPs, or rely on other sensing principles [8]. The majority of these devices is fabricated directly on the fibre tip, requiring complex and expensive/high-cost techniques such as electron beam/UV lithography and focused ion beam [9]. Such structures include metallic metamaterials (MMs), such as nanorods or nanostripes [10-13]. 
In this Letter, we demonstrate that it is possible to fabricate metallic nanowire $\mathrm{MM}$ films and mount them on the tip of a fibre, preserving its original optical properties with low-cost and high reproducibility. This means that we are not restricted to fabricate these structures directly on the fibre, allowing us to use standard low-cost self-assembly approaches, such as porous anodic aluminium oxide (AAO) templates filled with a metal. In this configuration the wires are aligned with the optical axis of the fibre and therefore can support SPPs along their elongated cylindrical surfaces [14] and can be used to develop high sensitivity optical sensors [15]. Another important advantage of a sensor based on this approach is that it would operate on reflection while standard fibre-optic SPP resonance sensors usually work on transmission, requiring the addition of a reflective element to the fibre to bounce the signal back. Therefore, our characterization of the MM device focuses on the reflected signal, rather than transmission.

This Letter is organized as follows: in Section 2 we summarize the fabrication process of the MM device, in Section 3 we overview the theoretical model used to simulate it, Section 4 describes the experimental methods used in the optical characterization, Section 5 analyses the results and, finally, Section 6 is devoted to the conclusions.

2 Fabrication The nanowire MMs were prepared following a standard bottom-up fabrication technique consisting of $\mathrm{Ag}$ electrodeposition inside a nanoporous AAO template serving as a scaffold. The AAO templates were synthesized in a two-step anodization process with $0.3 \mathrm{M}$ oxalic acid under an applied voltage of $40 \mathrm{~V}[16,17]$. This results in a nanoporous matrix of high aspect-ratio vertical cylinders, ordered by self-assembly in a hexagonal lattice with a natural porosity (defined as volume fraction of holes/pores) of $p \sim 0.10$ [18]. To produce Ag/AAO composites with different fill-ratios (volume fraction of metal), we used a controlled chemical etching process to widen the nanopores. The Ag nanowires were then grown inside the AAO matrix using a pulsed electrodeposition process (PED) [10]. Because not all nanowires grown by PED have the same height, the top surface of the MM films was subsequently submitted to a physical etching process $\left(\mathrm{Ar}^{+}\right.$ion beam milling) to increase the MM regularity [19]. As shown by the scanning electron microscopy (SEM) images of a produced MM film in Fig. 1, the fabrication process results in a nanowire polycrystal with a hexagonal lattice, where not all pores are completely filled with metal. The analysis of these images indicates that the MM has a porosity of $p=0.20 \pm 0.03$, a $\mathrm{Ag}$ fill-ratio of $f=0.14 \pm 0.03$, an interpore distance (lattice parameter) of $a=(96 \pm 9) \mathrm{nm}$, and a membrane thickness of $d=(5.6 \pm 0.1) \mu \mathrm{m}$.

The MM-fibre devices were constructed attaching the nanowire MM films to the tip of multimode silica optical fibres (Thorlabs FG550LEC) with 550/600 $\mu \mathrm{m}$ core/cladding diameters and numerical aperture of NA $=0.22$. The MM was carefully fixed and aligned perpendicular to the optical axis of the fibre using an in-house positioning sys-

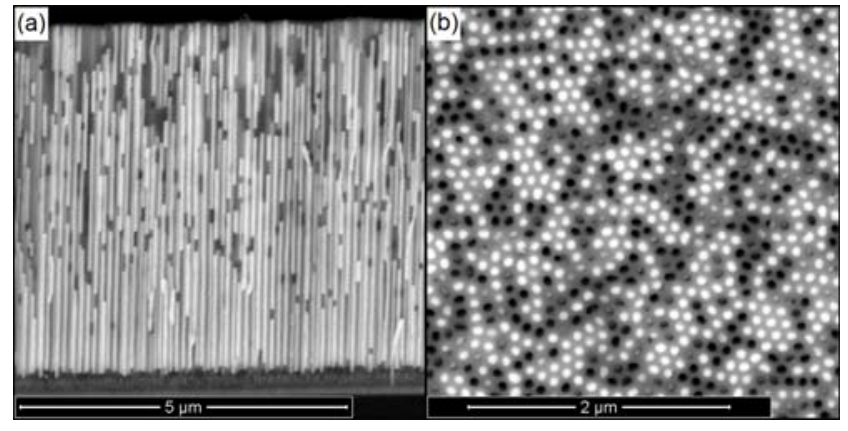

Figure 1 SEM images of the fabricated metamaterial: (a) cross section and (b) top view. Bright and dark spots correspond to Ag-filled and empty pores, respectively.

tem specifically designed for this purpose. The adhesion was ensured by an optical grade polymeric bonding agent used to promote the coupling of the fibre to the MM film, and guarantee the mechanical stability.

3 Model In this section we briefly review some of the basic physics of nanowire MMs and describe how the optical response of the MM-fibre device was simulated. Since not all the pores of the AAO template were filled with Ag, we have considered that there are two types of inclusions: $\mathrm{Ag}$ and air, with fill-ratios $f_{1}$ and $f_{2}=p-f_{1}$, respectively. Because the unit cell is sub-wavelength in the spectral range considered $(400-1000 \mathrm{~nm})$, the macroscopic optical properties of the MM can be calculated using MaxwellGarnett's homogenization theory $[20,21]$. Specifically, the effective permittivity is given by [21]

$$
\varepsilon_{\text {eff }}=\varepsilon_{\mathrm{h}} \frac{1+\eta \Sigma}{1-\Sigma}, \quad \Sigma=f_{1} \frac{\varepsilon_{1}-\varepsilon_{\mathrm{h}}}{\varepsilon_{1}+\eta \varepsilon_{\mathrm{h}}}+f_{2} \frac{\varepsilon_{2}-\varepsilon_{\mathrm{h}}}{\varepsilon_{2}+\eta \varepsilon_{\mathrm{h}}},
$$

where $\varepsilon_{\mathrm{h}}$ is the dielectric constant of the host medium $\left(\mathrm{Al}_{2} \mathrm{O}_{3}\right)$, and $\varepsilon_{1,2}$ are the electric permittivities of the two types of inclusions with fill-ratios $f_{1}$ and $f_{2}$, respectively. Moreover, $\eta$ is the screening factor which accounts for the shape of the inclusions ( $\eta=1$ for high aspect-ratio cylinders perpendicular to the electric field [22]). The values for $\varepsilon_{1}(\mathrm{Ag})$ and $\varepsilon_{\mathrm{h}}\left(\mathrm{Al}_{2} \mathrm{O}_{3}\right)$ were fitted from experimental data taken from reference [23]. The (complex) effective refractive index $\tilde{n}_{\text {eff }}=n_{\text {eff }}+i \kappa_{\text {eff }}=\left(\varepsilon_{\text {eff }}\right)^{1 / 2}$ is shown in Fig. 2 . Notice the peak around $400 \mathrm{~nm}$ which accounts for the strong absorption below $600 \mathrm{~nm}$ and the red colour observed in transmission in the Abstract figure.

The reflection spectrum of light on the MM-fibre device was simulated using the transfer-matrix method [24] for stratified optical media corresponding to the interfaces between the fibre core, the MM film and the transmission medium (air). In this model, the MM was replaced by an equivalent non-magnetic homogenous film (with the same thickness and refractive index $\tilde{n}_{\text {eff }}$ ) described by the characteristic matrix:

$$
\left[m_{i j}\right]=\left[\begin{array}{cc}
\cos \left(k_{0} \tilde{n}_{\mathrm{eff}} d\right) & \frac{-i}{\tilde{n}_{\text {eff }}} \sin \left(k_{0} \tilde{n}_{\mathrm{eff}} d\right) \\
-i \tilde{n}_{\mathrm{eff}} \sin \left(k_{0} \tilde{n}_{\mathrm{eff}} d\right) & \cos \left(k_{0} \tilde{n}_{\mathrm{eff}} d\right)
\end{array}\right]
$$




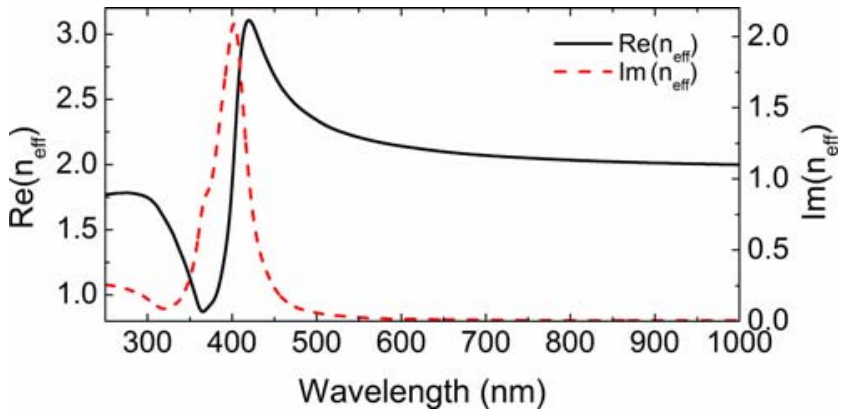

Figure 2 Effective refractive index obtained through the Maxwell-Garnett homogenization, where the real and imaginary parts are plotted as black-solid and red-dashed lines, respectively.

In Eq. (2), $k_{0}=2 \pi / \lambda$ is the free-space wavenumber $(\lambda$ is the wavelength in vacuum) and normal incidence is assumed (for $\mathrm{NA}=0.22$ the maximum incidence angle is $8.2^{\circ}$, which produces an almost identical reflectivity spectrum). The spectral reflectance of the film is given by

$$
R(\lambda)=|r|^{2}, \quad r=\frac{\left(m_{11}+m_{12} n_{\mathrm{t}}\right) n_{\mathrm{i}}-\left(m_{21}+m_{22} n_{\mathrm{t}}\right)}{\left(m_{11}+m_{12} n_{\mathrm{t}}\right) n_{\mathrm{i}}+\left(m_{21}+m_{22} n_{\mathrm{t}}\right)},
$$

where $n_{\mathrm{i}}$ and $n_{\mathrm{t}}$ are the refractive indices of the incidence (silica glass) and transmission (air) media, respectively. The results of the simulation are shown in Fig. 4(a).

4 Experimental methods The MM was characterized using reflection measurements before and after being mounted on the fibre using the experimental setups schematically depicted in Fig. 3(a) and (b), respectively. In the first setup [Fig. 3(a)] a fibre optic reflection probe (Avantes Reflection Probe) composed of six illumination fibres surrounding one collection fibre (each with $400 \mu \mathrm{m}$ core diameter and numerical aperture 0.22) was used to simultaneously obtain a uniform illumination and collection of the light scattered from the sample, under nearly normal incidence. We used a silver mirror as a reference to calibrate the reflection spectrum of the MMs and both a tungsten-halogen lamp (Avantes AvaLight-HAL) and a lightemitting diode (LED) as a light source covering a broad spectrum from $400 \mathrm{~nm}$ (where the LED is more stable than the lamp) to $2000 \mathrm{~nm}$. However, our measurements were limited by the working bandwidth of the spectrometer (Ocean Optics USB4000-VIS-NIR) spanning from 345$1050 \mathrm{~nm}$. In the second setup [Fig. 3(b)] we have replaced the reflection probe with a bifurcated optical fibre (Scansci ABF6020) connected to the MM-fibre device. In this case, we used as a reference the reflection signal from the core/air interface of a cleaved fibre. Figure 4(b) and (c) are obtained as the ratio between the reflection spectrum of the MM sample (b) or MM-fibre (c) and the reflection spectrum obtained from the corresponding reference, thus allowing to cancel out the intensity profile of the sources.

5 Results and discussion The experimental and simulated reflection signals for the exact same MM film are

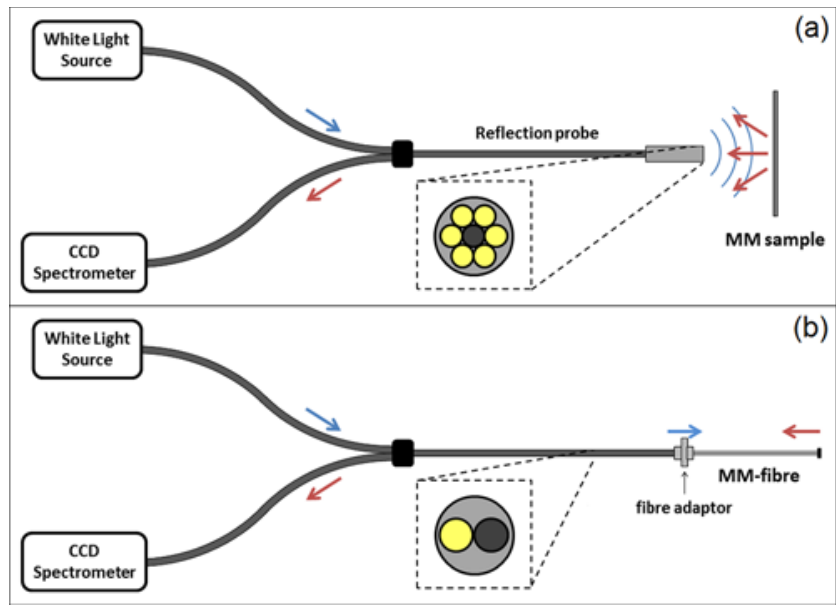

Figure 3 Schematic of the experimental setup used in the optical characterization of the (a) MM samples and the (b) MM-fibre devices. Insets: schematic of the cross section of the (a) optical fibre reflection probe and the (b) optical fibre coupler.

shown in Fig. 4 and are qualitatively similar. In particular, there is a very close match between the results from the MM sample [Fig. 4(b)] and the MM-fibre [Fig. 4(c)], indicating that the procedure used to fabricate the optical MM-fibre devices does not affect the optical properties of the MM. This means that the device can successfully excite the SPP modes in the metallic nanowires.

There is a red shift of the peak observed in Fig. 4(b) and (c) around $500 \mathrm{~nm}$ relative to the prediction of the Maxwell-Garnett model shown in Fig. 4(a), around $400 \mathrm{~nm}$, which considers an isolated wire. This type of shift has been attributed to the excitation and mutual interaction of the SPPs in the metallic nanostructures of the film, and has been confirmed by experiments [25] and simulations [26]. In our case, it is impossible to confirm the existence of SPP by rotating the polarization of the incident light because of the rotation symmetry of the system around the optical axis of the fibre. However, there is a qualitative consistency between the redshift observed in Fig. 4 and the demonstration in similar MM films with nanowires [25]. For wavelengths above $750 \mathrm{~nm}$ we can observe a modulation in all the reflection spectra, which is consistent with a Fabry-Pérot cavity formed by the upper and lower surfaces of the MM film. Below this wavelength, the imaginary refractive index of the MM is high and accounts for a large absorption (see Fig. 2) that prevents the interference of the multiple reflections. Furthermore, the separation $\Delta \lambda$ between consecutive peaks in the experimental data is in good agreement with the simulated results [Fig. 4(a)]: $\Delta \lambda \sim 33 \mathrm{~nm}$ and $\Delta \lambda \sim 34 \mathrm{~nm}, \quad$ respectively, around the central wavelength $\lambda_{0}=900 \mathrm{~nm}$. This is related to the refractive index $n$ and thickness $d$ of the film through [24]

$$
\Delta \lambda=\frac{\lambda_{0}^{2}}{2 n_{\mathrm{eff}} d+\lambda_{0}} .
$$




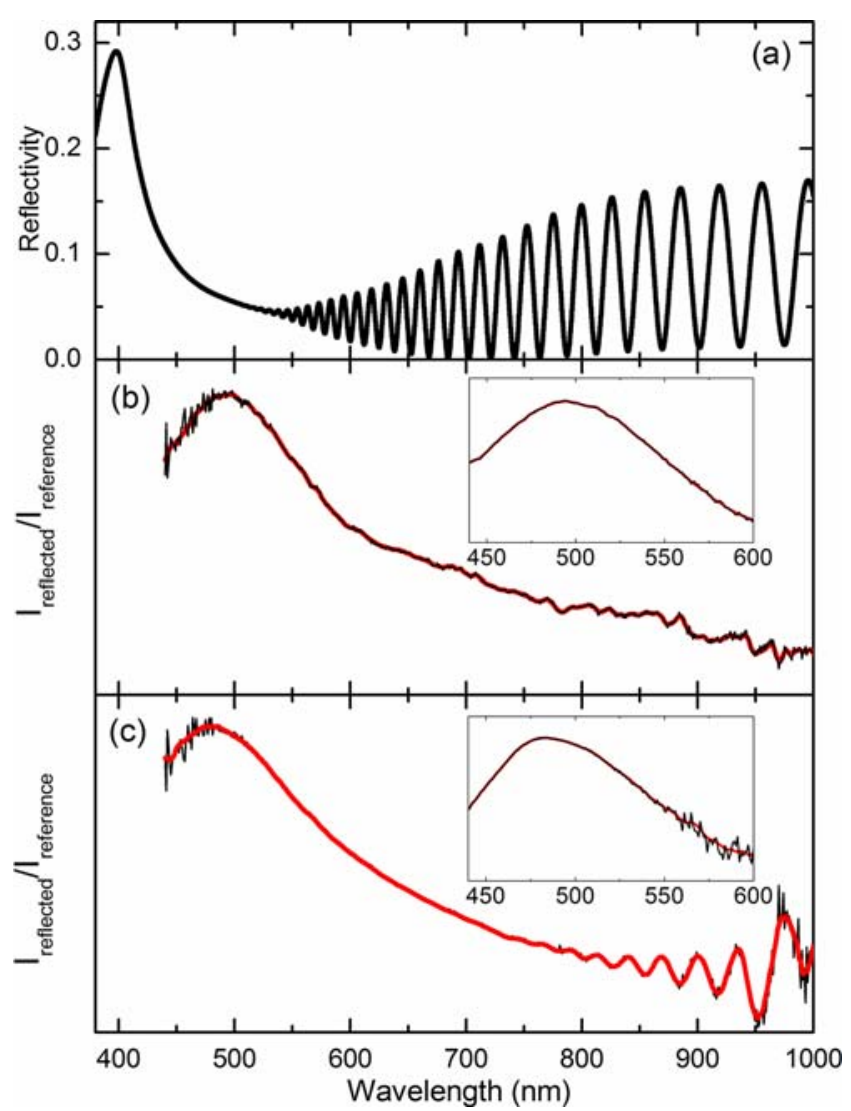

Figure 4 (a) Simulated reflection spectrum using the transfermatrix model. Experimental reflection spectra for the (b) MM sample and (c) MM-fibre device. Inset: measurements performed with an LED source.

Using Eq. (4) and $d=5.6 \mu \mathrm{m}$ we estimate an experimental value of $n_{\text {eff }} \approx 2.1$ at $\lambda_{0}=900 \mathrm{~nm}$, which is comparable to $n_{\mathrm{eff}} \approx 2.01$ from the Maxwell-Garnett model (Fig. 2).

6 Conclusions In this Letter, we have demonstrated that it is possible to fabricate nanowire MMs using lowcost bottom-up techniques and then mount them on optical fibre tips, while preserving their optical properties. Our results are compatible with the excitation of SPP in the nanowires. This would allow going beyond the traditional designs for SPR sensors in optical fibres, where the metallic films are placed on the tapered or side-polished fibre sections. As a consequence, the sensors with $\mathrm{MM}$ in the fibre tip will be inherently reflective. The technique demonstrated here is not limited to this specific nanowire MM. Instead, the optical properties of the MM can be customized by changing the metal and the geometries of the wires, or even choosing another membrane with completely different nanostructure. This opens the possibility of developing many different types of devices that make use of the myriad of exotic optical properties allowed by MMs. Both the fabrication and assembling techniques used are relatively simple, inexpensive, and reproducible, paving the way to industrial and large-scale production of photonic devices, such as sensors based on the integration of MMs in fibreoptic platforms.

Acknowledgements This work was developed within the framework of the project PG03109, financially supported by FCT - Fundação para a Ciência e a Tecnologia (Portuguese Foundation for Science and Technology) and project 275/2010, financially supported by CAPES - Coordenação de Aperfeiçoamento de Pessoal de Nível Superior (Brazilian Governmental Research Agency). A. Hierro-Rodríguez, J. M. Teixeira and M. P. Fernández-García acknowledge the support from FCT grants SFRH/ BPD/90471/2012, SFRH/BPD/72329/2010, and SFRH/BPD/ $87430 / 2012$, respectively. This work is also part-funded by the ERDF - European Regional Development Fund through the COMPETE Programme (operational programme for competitiveness) and by National Funds through the FCT within project "FCOMP-01-0124-FEDER-022701".

\section{References}

[1] A. Yu. Nikitin et al., Phys. Status Solidi RRL 4, 250 (2010).

[2] I. Iorsh et al., Phys. Status Solidi RRL 6, 43 (2012).

[3] J. Homola (ed.), Surface Plasmon Resonance Based Sensors (Springer-Verlag, Berlin, Heidelberg, 2006).

[4] A. K. Sharma et al., IEEE Sens. J. 7, 1118 (2007).

[5] E. Kretschmann and H. Raether, Z. Naturforsch. 23, 2135 (1968).

[6] R. C. Jorgenson and S. S. Yee, Sens. Actuators B 12, 213 (1993).

[7] I. Leite et al., Sens. Actuators B 160, 592 (2011).

[8] C.-L. Lee et al., IEEE Photon. Technol. Lett. 25, 833 (2013).

[9] M. Consales et al., ACS Nano 6, 3163 (2012).

[10] L. M. Custodio et al., Phys. Rev. B 85, 165408 (2012).

[11] S. Biswas et al., Nano Lett. 13, 2220 (2013).

[12] A. Olivieri et al., Phys. Status Solidi RRL 4, 283 (2010).

[13] B. Fan et al., Appl. Phys. Lett. 100, 111108 (2012).

[14] M. Bora et al., Nano Lett. 10, 2832 (2010).

[15] A. V. Kabashin et al., Nature Mater. 8, 867 (2009).

[16] H. Masuda and K. Fukuda, Science 268, 1466 (1995).

[17] D. C. Leitao et al., J. Phys. Chem. C 115, 8567 (2011).

[18] K. Nielsch et al., Nano Lett. 2, 677 (2002).

[19] C. T. Sousa et al., Nanotechnology 22, 315602 (2011).

[20] D. E. Aspnes, Am. J. Phys. 50, 704 (1982).

[21] W. Cai and V. Shalaev, Optical Metamaterials (Springer, 2010).

[22] W. T. Lu and S. Sridhar, Phys. Rev. B 77, 233101 (2008).

[23] E. D. Palik (ed.), Handbook of Optical Constants of Solids (Academic Press, Boston, 1995).

[24] M. Born and E. Wolf, Principles of Optics, 7th ed. (Cambridge University Press, 1999).

[25] P. K. Jain et al., Nano Lett. 7, 2080 (2007).

[26] A. O. Silva et al., arXiv: 1306.4150 [physics.optics]. 